\title{
Appel à contributions : L'environnement alimentaire au Canada
}

Diffuser cet appel sur Twitter

\section{Promotion de la santé et prévention des maladies chroniques au Canada : Recherche, politiques et pratiques}

Numéro spécial : L'environnement alimentaire au Canada

Sous la direction de Robert Geneau (rédacteur en chef, Agence de la santé publique du Canada) et Lana Vanderlee (rédactrice en chef invitée; Département des sciences nutritionnelles de l'Université de Toronto)

L’alimentation est un élément clé de la santé et les habitudes alimentaires sont en lien étroit avec le développement des maladies chroniques et de l'obésité. L'environnement alimentaire au sein duquel nous faisons nos choix alimentaires joue un rôle essentiel dans l'établissement de ces habitudes alimentaires et dans la qualité générale de l'alimentation. L'environnement alimentaire, englobant au sens large le contexte, les possibilités et les conditions physiques, économiques, politiques et socioculturelles ayant une influence sur nos choix alimentaires et sur notre état nutritionnel, peut favoriser une saine alimentation ou au contraire lui nuire.

L'environnement alimentaire au Canada ne favorise pas systématiquement les choix santé, ce qui se traduit par de mauvaises habitudes alimentaires à l'échelle de l'ensemble de la population. Au Canada comme dans d'autres pays, on s'intéresse de plus en plus à la manière dont on pourrait modifier l'environnement alimentaire afin de favoriser des comportements alimentaires plus sains.

C'est dans ce contexte que Promotion de la santé et prévention des maladies chroniques au Canada : Recherche, politiques et pratiques lance un appel à contributions portant sur l'environnement alimentaire au Canada. Tous les types d'articles sont les bienvenus. Nous sommes intéressés par des études touchant tous les aspects de l'environnement alimentaire (composition des aliments, étiquetage, promotion et commercialisation, fourniture et achats d'aliments, vente au détail, prix, commerce et investissements, etc.) et nous recherchons plus précisément des articles qui :

- caractérisent l'environnement alimentaire canadien actuel,

- examinent les répercussions des politiques et des interventions liées à l'environnement alimentaire en contexte canadien,

- synthétisent des données probantes sur l'état de l'environnement alimentaire au Canada.

Nous vous invitons à soumettre vos manuscrits à Journal_HPCDP-Revue_PSPMC@phac-aspc.gc.ca avant le 31 octobre 2016 en faisant explicitement référence à cet appel à contributions.

Pour de plus amples renseignements sur les lignes directrices relatives à la soumission d'articles et sur les types d'articles, veuillez consulter la page http://www.phac-aspc.gc.ca/publicat/hpcdp-pspmc/autinfo-fra.php 